\title{
High-Frequency Stimulation of the Subthalamic Nucleus Suppresses Oscillatory $\beta$ Activity in Patients with Parkinson's Disease in Parallel with Improvement in Motor Performance
}

\author{
Andrea A. Kühn, ${ }^{1,3}$ Florian Kempf, ${ }^{3}$ Christof Brücke, ${ }^{3}$ Louise Gaynor Doyle, ${ }^{1}$ Irene Martinez-Torres, ${ }^{1,2}$ Alek Pogosyan, ${ }^{1}$ \\ Thomas Trottenberg, ${ }^{3}$ Andreas Kupsch, ${ }^{3}$ Gerd-Helge Schneider, ${ }^{4}$ Marwan I. Hariz, ${ }^{1,2}$ Wim Vandenberghe, ${ }^{5}$ Bart Nuttin, ${ }^{6}$ \\ and Peter Brown ${ }^{1}$ \\ ${ }^{1}$ Sobell Department of Motor Neuroscience and Movement Disorders and ${ }^{2}$ Unit of Functional Neurosurgery, Institute of Neurology, London WC1N 3BG, \\ United Kingdom, Departments of ${ }^{3}$ Neurology and ${ }^{4}$ Neurosurgery, Charite Campus Virchow, Humboldt University, 13353 Berlin, Germany, and ${ }^{5}$ Division of \\ Experimental Neurology and ${ }^{6}$ Laboratory for Experimental Neurosurgery and Neuroanatomy, Katholieke Universiteit Leuven, Bus 50053000 Leuven, \\ Belgium
}

\begin{abstract}
High-frequency stimulation (HFS) of the subthalamic nucleus (STN) is a well-established therapy for patients with severe Parkinson's disease (PD), but its mechanism of action is unclear. Exaggerated oscillatory synchronization in the $\beta$ (13-30 Hz) frequency band has been associated with bradykinesia in patients with PD. Accordingly, we tested the hypothesis that the clinical benefit exerted by STN HFS is accompanied by suppression of local $\beta$ activity. To this end, we explored the after effects of STN HFS on the oscillatory local field potential (LFP) activity recorded from the STN immediately after the cessation of HFS in 11 PD patients. Only patients that demonstrated a temporary persistence of clinical benefit after cessation of HFS were analyzed. STN HFS led to a significant reduction in STN LFP $\beta$ activity for $12 \mathrm{~s}$ after the end of stimulation and a decrease in motor cortical-STN coherence in the $\beta$ band over the same time period. The reduction in LFP $\beta$ activity correlated with the movement amplitude during a simple motor task, so that a smaller amount of $\beta$ activity was associated with better task performance. These features were absent when power in the 5-12 $\mathrm{Hz}$ frequency band was considered. Our findings suggest that HFS may act by modulating pathological patterns of synchronized oscillations, specifically by reduction of pathological $\beta$ activity in PD.
\end{abstract}

Key words: DBS; mechanism; $\beta$ oscillation; STN; Parkinson's disease; motor

\section{Introduction}

The high-frequency stimulation (HFS) of either the subthalamic nucleus (STN) or internal globus pallidus (GPi) is a highly effective treatment for Parkinson's disease (PD). However, the mechanism by which stimulation at $100-180 \mathrm{~Hz}$ has its therapeutic effects remains unclear. Work over the last decade has suggested that exaggerated synchronization of basal ganglia neurons may underpin Parkinsonian motor deficits (Gatev et al., 2006; Uhlhaas and Singer, 2006; Hammond et al., 2007) leading to the hypothesis that HFS may suppress pathological synchronization (Brown and Eusebio, 2008). In nonhuman primates made parkinsonian through injection of 1-methyl-4-phenyl-1,2,3,6tetrahydropyridine, synchronization tends to occur at frequen-

\footnotetext{
Received Jan. 22, 2008; revised April 7, 2008; accepted April 8, 2008.

This work was supported by a fellowship from the National Parkinson Foundation (A.A.K.), the Parkinson's Appeal (M.I.H.), and the Medical Research Council of Great Britain (P.B.).

Correspondence should be addressed to Prof. Andrea A. Kühn, Department of Neurology, Charité, University Medicine Berlin, Campus Virchow Klinikum, Augustenburger Platz 1, 13353 Berlin, Germany. E-mail: andrea.kuehn@charite.de.

DOI:10.1523/JNEUROSCI.0282-08.2008

Copyright $\odot 2008$ Society for Neuroscience $\quad$ 0270-6474/08/286165-09\$15.00/0
}

cies < $15 \mathrm{~Hz}$ (Bergman et al., 1994; Nini et al., 1995), and extracellular recordings of neuronal discharges during HFS demonstrate a suppression of synchrony at these lower frequencies (Meissner et al., 2005). In patients with PD, oscillatory synchronization tends to occur at frequencies of $\sim 20 \mathrm{~Hz}$, in the so-called $\beta$ band (Hammond et al., 2007). Generally this synchronization has been inferred from oscillations in local field potentials (LFPs), and several lines of evidence support the use of such activity as a surrogate measure of neuronal synchrony in the basal ganglia (Levy et al., 2002; Brown and Williams, 2005; Kühn et al., 2005; Weinberger et al., 2006).

It has proven very difficult to record LFP activity during stimulation using the same implanted macroelectrode used for clinical HFS, because stimulation-related artifacts are approximately three orders of magnitude bigger than LFP oscillations. Two approaches remain, whereby the suppression of $\beta$ activity by HFS can be tested in patients. First, LFP activity can be recorded from $\mathrm{GPi}$, a major output stage of the basal ganglia, during subthalamic deep brain stimulation (DBS) in rare patients in whom both nuclei are implanted on the same side of the brain. To date, two PD patients have been reported in whom HFS in the region of the 
STN lead to suppression of $\beta$ activity in GPi (Brown et al., 2004). Second, one can take advantage of the temporary persistence of the clinical effects of HFS in some patients (Temperli et al., 2003) to record LFP activity immediately after the cessation of stimulation. Here, the results are conflicting. There is one preliminary report of suppression of $\beta$ LFP power in STN after STN HFS in two patients (Wingeier et al., 2006), but this was not corroborated in another study (Foffani et al., 2006). Importantly, all the above studies failed to correlate changes across the frequency spectrum with improvements in behavioral performance.

Here, we test the hypothesis that HFS of STN suppresses enhanced $\beta$ activity in $\mathrm{PD}$, and that this effect is correlated with the clinical improvement in motor symptoms in those patients.

\section{Materials and Methods}

\section{Patients and surgery}

Ten patients with Parkinson's disease (three female; age, $60 \pm 12$ years, mean \pm SD; disease duration, $10 \pm 4$ years) who underwent bilateral implantation of deep brain electrodes in the STN participated in the study, together with an additional patient who underwent simultaneous bilateral implantation of macroelectrodes in STN and GPi (case 11) because of severe dyskinesia. The clinical details are summarized in Table 1. Patients were operated at the Charité University Hospital, Campus Virchow (Berlin, Germany), the National Hospital for Neurology and Neurosurgery (London, UK), and the University Hospital in Leuven. All patients took part with informed consent, which was approved by the local ethics committee in accordance with The Code of Ethics of the World Medical Association (Declaration of Helsinki, 1967).

The surgical procedure has been reported in detail previously (Kühn et al., 2005). The DBS-electrode used was model 3389 (Neurologic Division, Medtronic) with four platinium-iridium cylindrical surfaces (1.27 $\mathrm{mm}$ diameter and $1.5 \mathrm{~mm}$ length) and a contact-to-contact separation of $0.5 \mathrm{~mm}$. The intended coordinates at contact 0 were $10-12 \mathrm{~mm}$ from the midline, $0-3 \mathrm{~mm}$ behind the midcommisural point, and 3-4 $\mathrm{mm}$ below the anterior commissure-posterior commissure line. Adjustments to the intended surgical coordinates were made according to the direct visualization of STN on individual preoperative stereotactic $\mathrm{T}_{2}$-weighted magnetic resonance imaging (MRI) (Hariz et al., 2003) and, in cases $1-4$ from Berlin and case 10 from Leuven, intraoperative microelectrode recordings with TREC-scanner electrophysiological neuronavigation system using a tetrode (Thomas Recording) and Medtronic Leadpoint microelectrode recording system (Medtronic), respectively. Intraoperative electrical stimulation and immediate postoperative stereotactic MRI were performed in all patients (except for case 10) to confirm targeting. In the latter, the position of the DBS-macroelectrode was confirmed by an anteroposterior and lateral x-ray in stereotactic conditions after implantation and fixation of the electrodes. Postoperative imaging confirmed that at least two contacts of each macroelectrode were within the STN (see Table 1). After surgery, patients had an overall improvement of $54.9 \pm 5.0 \%(n=9$; cases $1-6,8,9,11$; mean \pm SE) using the Unified PD Rating Scale (UPDRS) motor score during the standard clinical settings of continuous DBS without medication compared with UPDRS scores off stimulation. Postoperatively, antiparkinsonian medication was reduced by $32.4 \pm 11.1 \%$ after converting dopaminergic drugs to levodopa-equivalent dose $(1 \mathrm{mg}$ of pergolide $=1 \mathrm{mg}$ of pramipexole $=2$ $\mathrm{mg}$ of cabergoline $=4 \mathrm{mg}$ of ropinirole $=100 \mathrm{mg}$ of levodopa with a D-decarboxylase inhibitor).

\section{Paradigm and recordings}

The postoperative recording sessions took place $2-5 \mathrm{~d}$ after the electrode implantation. All patients were examined after overnight withdrawal of antiparkinsonian medication. Initially, we defined the threshold for clinically effective stimulation and/or occurrence of side effects of HFS on each side using an external stimulator (DualScreen model 3628; Medtronic) with a pulse width of $60 \mu$ s and stimulation frequency of 130 $\mathrm{Hz}$. To limit the time of clinical test stimulation, we always used contacts $0-$ and $2+$, because these contacts most likely would include the target area (estimated at the center of contact 1 from stereotactic planning and

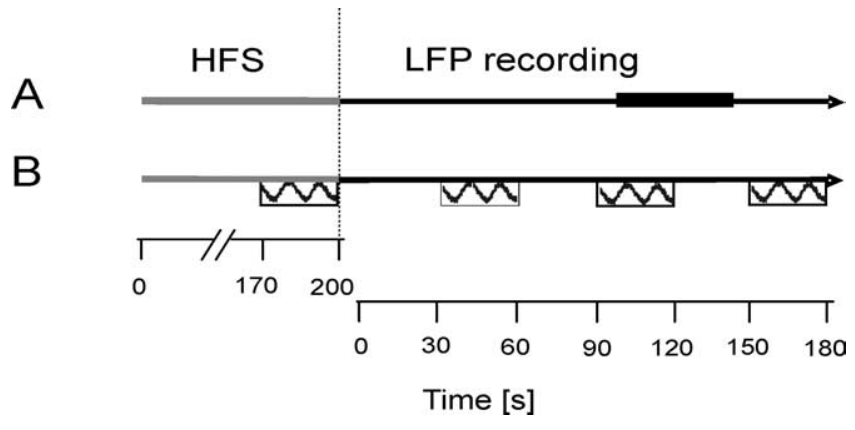

Figure 1. Schema of the experimental paradigm with LFP recordings at rest $(\boldsymbol{A})$ and motor task (B). The horizontal gray line represents the $200 \mathrm{~s}$ time period of HFS of the STN during one cycle of the experiment. The horizontal black line represents the time period of LFP recordings. The thick black line in $\boldsymbol{A}$ marks the period taken as baseline (100-140 s), and the boxes in $\boldsymbol{B}$ represent hand movements (interleaved by $30 \mathrm{~s}$ rest). Hand movements were performed once during HFS and three times after the end of HFS (post-HFS 1-3).

microrecording results), except for those cases where clinical side effects occurred at stimulation amplitudes below $1.5 \mathrm{~V}$ without clinical effect on motor symptoms. In the latter cases, contact 1 was used instead of contact 0 (cases 7 and 9) (for details, see Table 1). Rigidity and akinesia (repetitive opposing movements of index finger and thumb) were assessed and subjectively rated according to the UPDRS (motor score item 22 and 23) by an experienced clinician (A.A.K. or T.T.) to define the amplitude for clinically effective HFS. Stimulation amplitude was always kept below the threshold for side effects. In 8 of 22 sides (36\%), contact 1 , which was within the electrical field during bipolar stimulation (at contact $0-, 2+$ ), was used for long-term therapeutic stimulation and all but one of the remaining contact pairs (13 of 14) chosen for bipolar stimulation included a contact that was used postoperatively. The mean stimulation amplitude during bipolar stimulation $(3.7 \pm 0.2 \mathrm{~V})$ was higher compared with postoperative long-term HFS with monopolar stimulation $(2.4 \pm$ $0.3 \mathrm{~V} ; p<0.001$ ), which is consistent with the notion that bipolar stimulation amplitudes are usually higher to reach a similar energy for effective stimulation (Volkmann et al., 2006).

During the experiment, patients were seated comfortably in an armchair. The experimental protocol consisted of two parts (Fig. 1, schema). First, bilateral HFS was performed for $200 \mathrm{~s}$ followed by the recording of local field potentials from the STN area for $150 \mathrm{~s}$ once stimulation had been discontinued. This cycle was repeated twice in case 6 , in whom fatigue and discomfort obviated more trials, thrice in four cases and four times in five patients, with the patient at rest. Second, sessions of $200 \mathrm{~s}$ of HFS were combined with the performance of a motor task with the contralateral hand. Patients were asked to perform fast and largeamplitude alternating pronation and supination of the wrist using the more affected hand (or both hands in cases 1 and 8, but tested separately). Movement speed and angular degree (amplitude) of the hand movements were registered using a custom-made device ("rotometer"). The movements were performed for $30 \mathrm{~s}$ each with the first movement occurring from $170-200 \mathrm{~s}$ of the HFS period (i.e., just before cessation of stimulation). Three subsequent movement periods followed after HFS had been stopped and were interleaved by $30 \mathrm{~s}$ of rest (hereafter named as post-HFS 1-3). The same movement paradigm was repeated in two of the patients during continuous HFS over the entire test period of $350 \mathrm{~s}$ to test for potential effects on movement speed or amplitude related to fatigue in those patients.

The duration of stimulation and LFP recording was chosen to obtain reliable clinical effects, allow averaging and yet limit the duration of the experiment so as to avoid fatigue and discomfort. EEG over motor areas was recorded continuously during the entire experiment, and LFPs in the STN area were recorded as soon as possible after the end of HFS in all trials (except for movement trials in patient 10). The gap between the end of HFS and the start of LFP recordings was between 2 and $6 \mathrm{~s}$ because of the delay for switching from stimulation to recording and for stabilization of the amplifiers.

LFP activity was recorded at rest and during task performance bipo- 


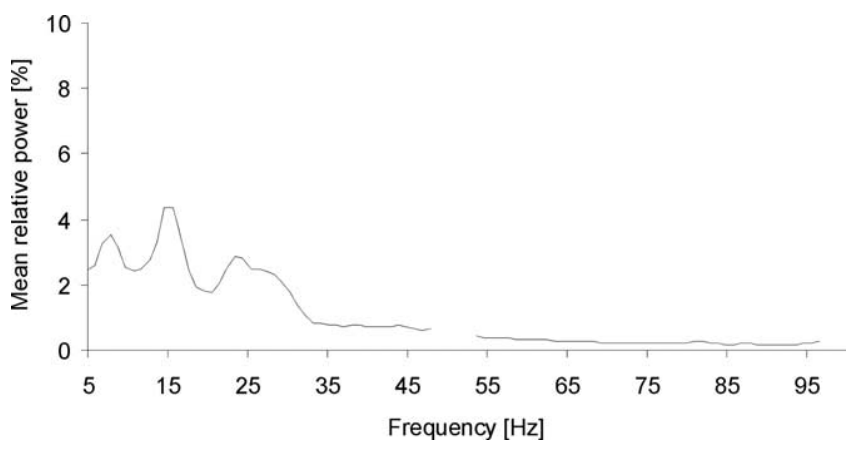

Figure 2. Power spectrum averaged across all patients off medication, estimated using the contact pairs displaying the maximum $\beta$ power per macroelectrode during the baseline period from 100 to $140 \mathrm{~s}$ after the end of HFS. Power is expressed as a percentage of total power in the $5-100 \mathrm{~Hz}$ range. Note that there is no distinct peak of activity above $30 \mathrm{~Hz}$ off medication.

larly from the four adjacent contacts of each DBS-electrode (contacts 01, 12 , 23). Signals were sampled at $625 \mathrm{~Hz}(n=1)$ or $1000 \mathrm{~Hz}(n=9)$, bandpass filtered at $1-250 \mathrm{~Hz}$, and amplified $(50,000 \times)$ using a custommade, $9 \mathrm{~V}$ battery-operated, portable high-impedance amplifier in Berlin and a D150 amplifier (Digitimer) in London. Signals were digitized through a 1401 A-D converter (Cambridge Electronic Design) recorded onto a computer using Spike2 software (Cambridge Electronic Design) and monitored on-line. In case 10, signals were amplified and pass-band filtered between 1.5 and $200 \mathrm{~Hz}$ (sampling rate, $1200 \mathrm{~Hz}$ ) using the Biopotential Analyzer Diana (Sechenov Institute of Evolutionary Physiology and Biochemistry, St Petersburg, Russia). EEG activity over motor areas was recorded (with the same filter and amplification as detailed for LFPs) using silver/silver chloride electrodes over Cz, C3, and/or C4, referenced to the ears, in those seven patients in whom surgical dressings allowed such recordings.

\section{Analysis}

Recordings at rest. Data were interpolated to a common sampling rate of $1 \mathrm{kHz}$. The first analysis step was to calculate spectral power changes for all contact pairs for a period of $0-140 \mathrm{~s}$ of rest recordings immediately after HFS. Spectra were estimated using the discrete Fourier transform as outlined by Halliday et al. (1995) and by Brown (2000). STN LFPs were assumed to be realizations of stationary, zero-mean time series and to satisfy a mixing condition, whereby sample values widely separated in time were independent (Brillinger, 1981). Records were divided into a number of sections of equal duration with a block size of 1024 data points, affording a frequency resolution of $\sim 1 \mathrm{~Hz}$. Spectra were estimated by averaging across sections, and a Hanning window filter was used. Blocks of $1 \mathrm{~s}$ were shifted by $500 \mathrm{~ms}$ until the whole record length had been analyzed (using a modified Spike2 script). Data for all sessions in each patient were averaged. Power changes over time were defined as the percentage power decrease or power increase in relation to a baseline period. The latter was chosen as a period at the end of each recording from 100 to $140 \mathrm{~s}$ post-HFS offset. An alternative would have been to select a baseline period before HFS onset, but then this would have preceded our period of interest by $>300 \mathrm{~s}$, and may have been less representative because of possible baseline drifts related to the changes in pharmacological state, fatigue, and attentional levels during the experiment. The use of a control period closer in time to the period of interest might therefore be deemed more valid. The mean relative power at baseline period was calculated as a proportion of the total power in the frequency range between 5 and $98 \mathrm{~Hz}$ (excluding activity at $50 \pm 2 \mathrm{~Hz}$ and $>98 \mathrm{~Hz}$ for mains noise and its harmonic, as well as activity $<5 \mathrm{~Hz}$ prone to movement artifact). Distinct spectral peaks were only found in the frequency range from 5 to $30 \mathrm{~Hz}$, which included on average $76 \%$ of the total power (Fig. 2). Based on the hypothesis that $13-30 \mathrm{~Hz}$ activity may promote bradykinesia and rigidity and should therefore be suppressed by effective HFS, we picked the contact pair that displayed the maximum $\beta$ activity during the baseline period for additional analysis (Table 1). This approach was also used for the two cases reported by Wingeier et al.
(2006). Contact pairs with the greatest $\beta$ power had at least one contact in STN in $92 \%$ ( 11 of 12 sides) as confirmed by postoperative imaging.

Time-evolving mean percentage power changes for each selected contact pair were estimated for the side contralateral to tested hand movements and averaged across patients. For visualization, time-frequency plots of spectral power were smoothed using a sliding average of $1.5 \mathrm{~s}$ window size. Group data of time-evolving power spectra were analyzed by means of the Wilcoxon signed rank test (i.e., in each time-frequency bin of matrices, we tested whether the median of the individual changes was different from baseline). This nonparametric approach was used as matrix values that were not distributed normally (Kolmogorov-Smirnov test). The resulting matrix was displayed with each bin represented by its respective $Z$ score, and the direction of change (i.e., the prefix) for each bin was defined according to the median of the populations tested in the respective bin (Kempf et al., 2007). The matrix was thresholded at a $Z$ score of \pm 1.9 .

In a second step of analysis, frequency bands of interest were selected for additional quantitative analysis. We chose the $13-30 \mathrm{~Hz}$ band based on our hypothesis of HFS-induced suppression of $\beta$ activity and in accordance with previous observations of $\beta$ power changes after HFS (Wingeier et al., 2006) and the $5-12 \mathrm{~Hz}$ band to test the frequencyspecific effects of HFS on oscillatory STN LFP activity. In addition, individual peaks within the $13-30 \mathrm{~Hz}$ range were defined from baseline LFP spectra. A $7 \mathrm{~Hz}$ wide $\beta$ band, including the peak frequency, and $3 \mathrm{~Hz}$ to either side of the peak was used for subanalysis of individual $\beta$ activity. The mean power change over time in these bands was normally distributed (Kolmogorov-Smirnov test) and compared with power in the baseline period using Student's $t$ test, and $p$ values below 0.05 were considered significant if present over at least three consecutive time points.

Coherence analysis between STN-LFP and EEG over cortical areas was performed in seven patients (eight STN) at rest using EEG over the ipsilateral motor cortex $(\mathrm{C} 3 / \mathrm{C} 4 ; n=6)$ or midline $\mathrm{EEG}(\mathrm{Cz}, n=2$ cases, in whom recordings over $\mathrm{C} 3 / \mathrm{C} 4$ were obviated by the surgical dressing). Coherence over $5-12 \mathrm{~Hz}, 13-30 \mathrm{~Hz}$, and individual $\beta$ frequencies were compared over two time periods $0-12 \mathrm{~s}$ after the end of HFS and at baseline from 128 to $140 \mathrm{~s}$ after HFS. The post-HFS period was defined based on the time course of $p$ values from direct comparison of band power between post-HFS and baseline (see above), and a time period of corresponding duration at baseline was chosen to allow for paired comparison of data. Coherence was calculated with a block size of 1024 that afforded a frequency resolution of $\sim 1 \mathrm{~Hz}$ and a time epoch of $1 \mathrm{~s}$ using a modified Spike 2 script.

Recordings during performance of the motor task. The mean movement amplitude for each trial in each patient was calculated as root mean square (RMS) amplitude over a time period of $20 \mathrm{~s}$ and expressed as percentage change from the last (fourth) movement that was performed in each cycle. Data from each movement (of 1-4) were separately averaged across sessions for each patient, and only patients that showed a decrease of at least $10 \%$ of movement amplitude between two subsequent (20 s) blocks of post-HFS movements were included in the study.

In addition, we assessed the relationship between STN-LFP power and performance of the movement task in individual patients ( $n=11$ sides). Three consecutive periods of $20 \mathrm{~s}$ of STN-LFP before each of the three post-HFS hand movements were used to calculate the mean power in the 5-12 Hz, the $13-30 \mathrm{~Hz}$ band, and the individual $\beta$ band. Power was normalized and expressed as percentage of power recorded before the last movement trial and correlated with the mean relative RMS amplitude of the movement.

The statistical analysis was conducted using SPSS v12 (SPSS) and Matlab (MathWorks). Because the data were not always normally distributed as confirmed by the one-sample Kolmogorov-Smirnov test, we used nonparametric statistics when necessary (as described in Results). Otherwise, repeated-measures ANOVAs were performed as detailed in Results, and post hoc two-tailed, paired Student's $t$ tests (using step-wise correction for multiple comparisons) were used to confirm relevant power changes. Correlations were performed using bivariate correlation for parametric data (Pearson correlation coefficient). Means \pm SEM are given below. 


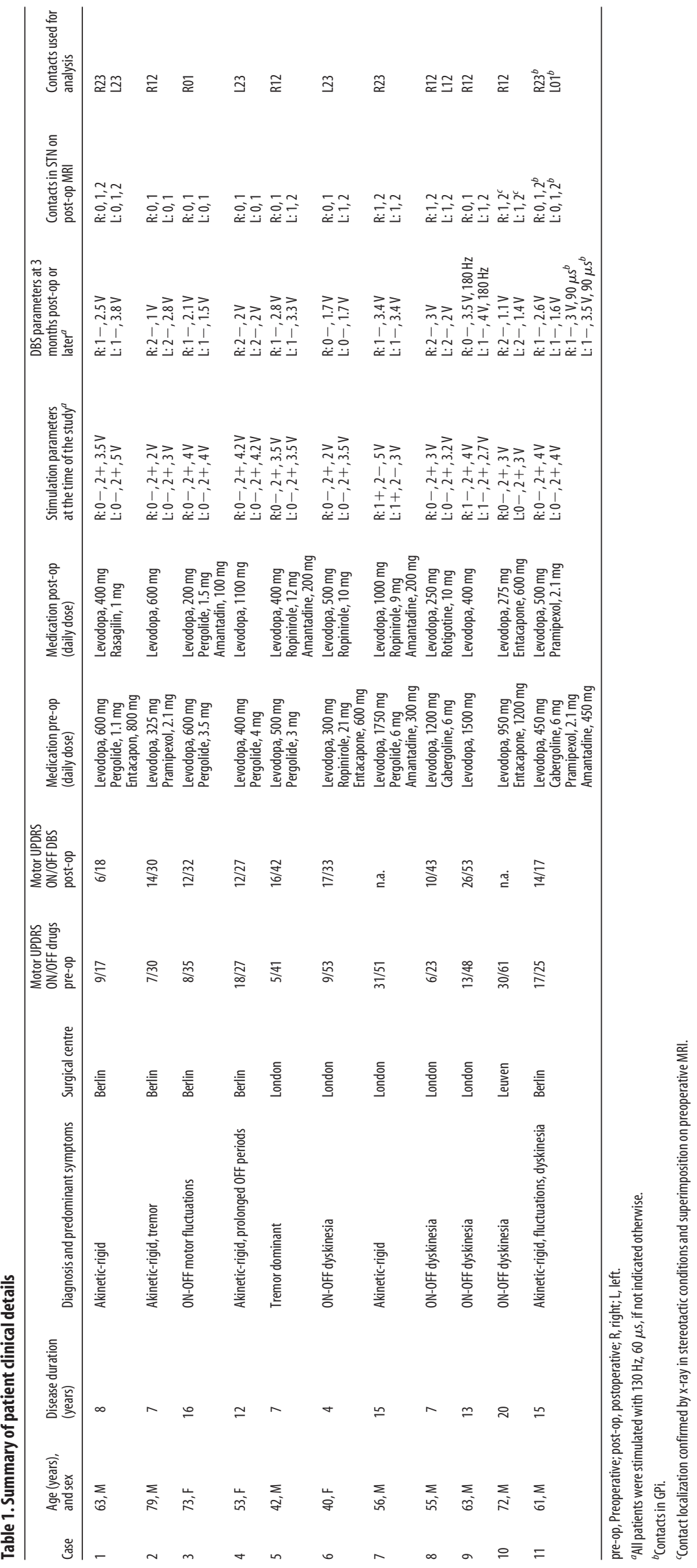



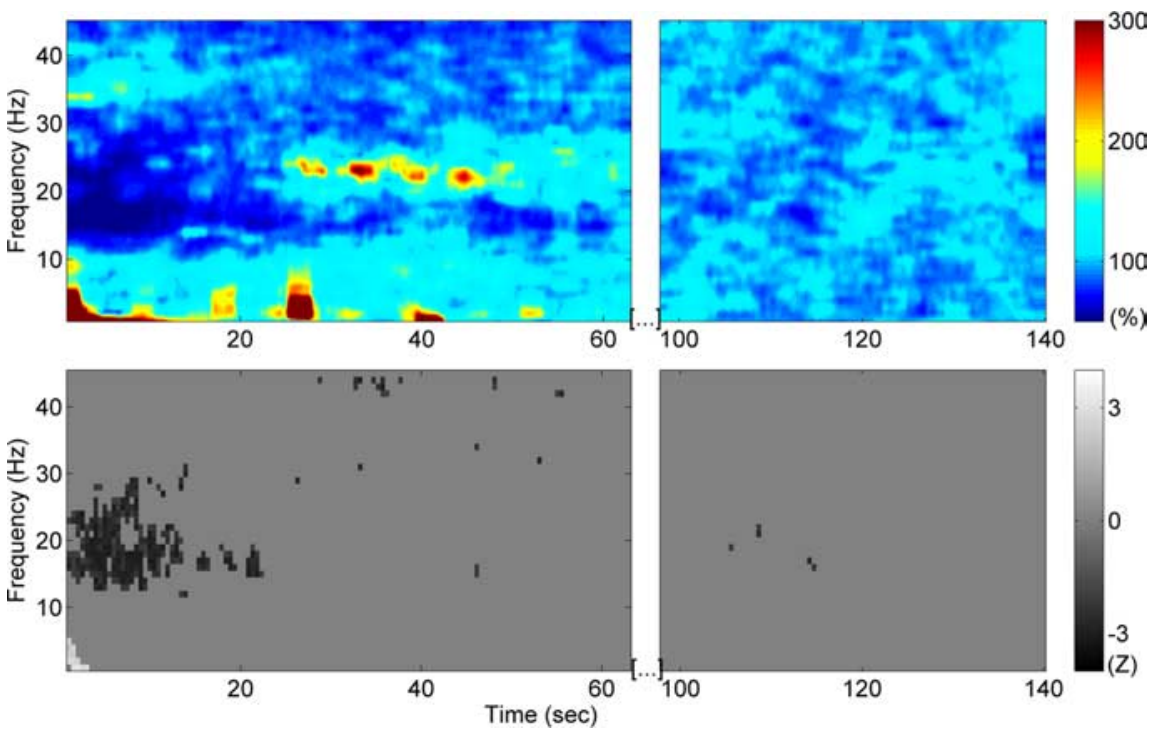

Figure 3. Time-frequency plot showing LFP activity after the end of HFS at time 0 expressed as a percentage change from baseline activity taken from 100 to $140 \mathrm{~s}$ after cessation of stimulation. Top, Mean relative power across 12 sides shows a suppression of $\beta$ activity after HFS (blue colors) followed by a relative increase in $\beta$ power (red colors) that was observed in some patients. Bottom, Corresponding $Z$ scores as determined by Wilcoxon's signed rank test thresholded at $Z= \pm 1.9$, confirming significant $\beta$ suppression that persists for $\sim 20$ s after the end of HFS.

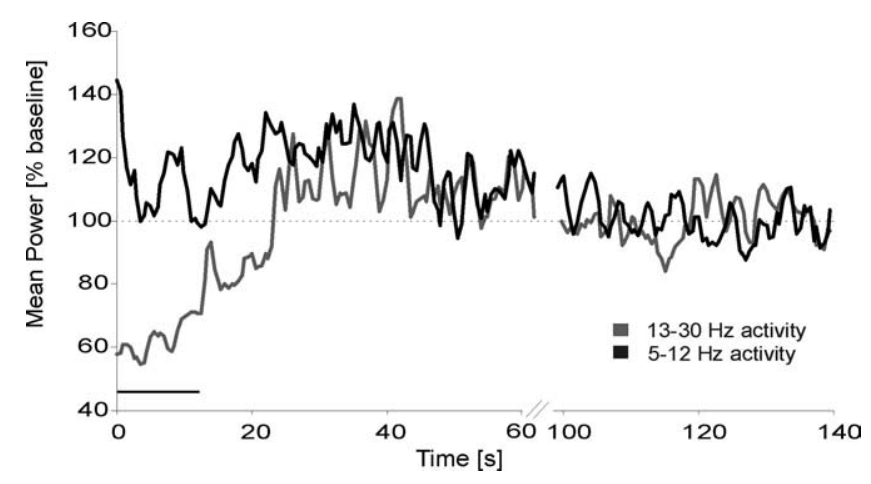

Figure 4. Time course of mean low-frequency $(5-12 \mathrm{~Hz}$; black line) and mean $\beta(13-30 \mathrm{~Hz}$; gray line) power after the end of HFS expressed as a percentage change from baseline activity (100-140 s after HFS). Time-evolving $p$ value (Student's $t$ test) revealed a significant suppression of $\beta$ activity for 12 s after the end of HFS compared with baseline (significant time period is indicated by horizontal black line). Note that power suppression after HFS is frequency specific, and no significant change occurred in the $5-12 \mathrm{~Hz}$ band.

\section{Results}

\section{Time course of STN LFP activity after HFS}

The time-frequency plot of mean relative power changes showed a decrease in oscillatory activity after the end of HFS that persisted for $\sim 20 \mathrm{~s}$ and was most pronounced in the $\beta$ frequency range (Fig. 3, top). This was followed by a temporary and inconsistent rebound in $\beta$ activity (Fig. 3, top). Figure 3 (bottom) shows the significant $Z$-score-transformed group data of mean power and confirms suppression of $\beta$ activity after the end of HFS.

Time-evolving power changes were evaluated for both frequency bands of interest. No significant change from baseline activity was revealed for the $5-12 \mathrm{~Hz}$ (Student's $t$ test), but a significant and continuous decrease in $\beta$ power $(13-30 \mathrm{~Hz})$ of up to $40 \%$ of baseline activity (100-140 s) was found for $12 \mathrm{~s}$ after the end of HFS (Fig. 4). The suppression of $\beta$ power was even more pronounced when individual $\beta$ bands were used for analysis (degree of suppression, up to 60\%) (data not shown). This

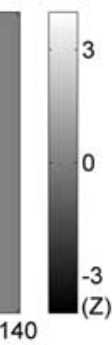

general picture was seen in all individual patients, although the time course of suppression of $\beta$ power varied. For individual data, the recurrence of $\beta$ activity was measured as an increase in $\beta$ power that reached the $95 \%$ confidence limits of baseline activity for at least three consecutive time points after a period of sustained suppression after HFS. The mean period of $\beta$ suppression was $24.0 \mathrm{~s} \pm 4.3$ (range, 9.5-55 s). An individual example is shown in Figure 5.

\section{Movement amplitude and its relationship to $\boldsymbol{\beta}$ activity}

One of the prerequisites for our study was the temporary persistence of the clinical effect of therapeutic HFS on bradykinesia and rigidity after the end of HFS (Temperli et al., 2003). Assessment of RMS movement amplitude in our patients confirmed a significantly higher movement amplitude during HFS that was still larger after the end of HFS but showed a progressive decrease over time (Friedman test, $\chi^{2}=$ 20.167, $p<0.001$; and post hoc Wilcoxon signed rank, $p<0.01$ ) (Fig. 6). No decrease in movement amplitude over time was revealed in two patients that performed the movement task during continuous HFS.

Additional assessment of the relationship between STN-LFP power changes after HFS and performance of the movement task poststimulation in individual patients revealed a significant negative correlation for mean relative movement amplitude and mean relative $\beta$ power (expressed as percentage change from the last movement amplitude and power in each session, respectively; $r=-0.499 ; p=0.018)$. Similarly, a significant negative correlation was found for individual $\beta$ power and movement amplitude $(r=-0.555 ; p=0.007)$ (Fig. 7), but there was no correlation with $5-12 \mathrm{~Hz}$ power $(r=0.108$; $p=0.634)$. Therefore, the relative improvement in motor performance was correlated with a decrease in relative $\beta$ power.

\section{EEG-STN LFP coherence after HFS}

Coherence between STN-LFP and EEG over motor cortical areas at low frequency $(5-12 \mathrm{~Hz})$ and individual $\beta$ frequency were compared over two time periods: (1) 0-12 s after the end of HFS and (2) at baseline from 128 to $140 \mathrm{~s}$ after HFS using a two-way repeated measure ANOVA with factors FREQUENCY (5-12 Hz and individual $\beta$ band) and TIME (postHFS and baseline). No main effect for FREQUENCY or TIME was found, but there was a significant interaction between the two factors $\left(F_{(1,7)}=6.554 ; p=0.038\right)$. Post hoc paired Student's $t$ test revealed a significantly smaller coherence for individual $\beta$ frequency after the end of HFS $(0.097 \pm 0.01)$ compared with baseline values $(0.13 \pm 0.01 ; p=0.008)$ (Fig. 8 ). No difference between post-HFS and baseline coherence values was revealed for low-frequency activity (post-HFS coherence, $0.15 \pm 0.02$; baseline, $0.13 \pm 0.01 ; p=0.366$ ). Coherence at $\beta$ frequency was significantly smaller compared with low frequency after the end of HFS $(p=0.017)$ but similar at baseline period $(p=0.6)$. Likewise, we found a trend for a smaller coherence within the broad $\beta$ band (13-30 

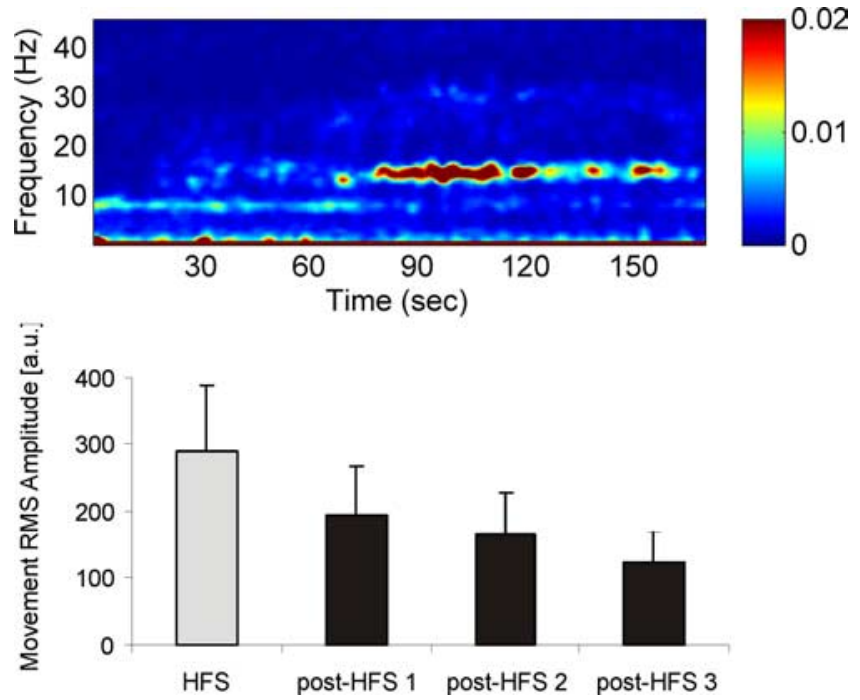

Figure 5. Top, Time-frequency plot of power changes after HFS in case 1. The average of three sessions of 200 s HFS and subsequent LFP recordings is shown. Note that $\beta$ activity $\sim 15$ $\mathrm{Hz}$ is completely suppressed for $\sim 30 \mathrm{~s}$ and then slowly recurs over the following $40 \mathrm{~s}$, paralleling a decrease in movement amplitude (bottom). Bottom, Mean RMS movement amplitude from three separate sessions in case 1 . The movements were performed for $30 \mathrm{~s}$ each with the first movement occurring just before cessation of HFS (gray column). The three subsequent movement periods followed after HFS had been stopped (post-HFS 1-3; black columns) and were interleaved by $30 \mathrm{~s}$ of rest.

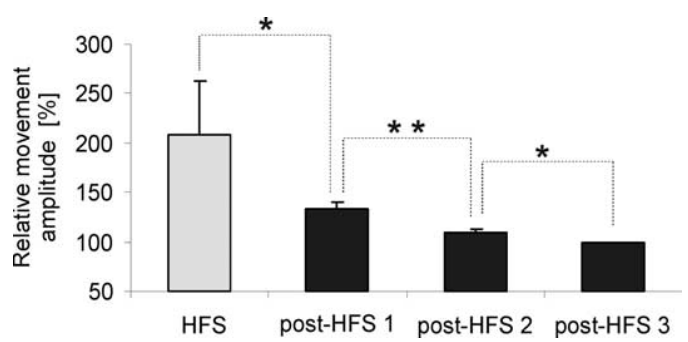

Figure 6. RMS movement amplitude averaged across all sessions in all patients ( $n=12$ sides) during (gray) and post-HFS (black) expressed as a percentage change from the amplitude of the last (fourth) movement. Movement amplitude is highest during HFS and shows a significant stepwise decrease over time after the end of HFS (Wilcoxon signed rank test with stepwise correction for multiple comparisons). ${ }^{*} p<0.05 ;{ }^{* *} p<0.01$.

$\mathrm{Hz}$ ) post-HFS compared with baseline ( $p=0.06$; paired Student's $t$ test).

\section{Pallidal LFP power during STN HFS in the patient with implantation of STN and GP}

In one additional patient (case 11), macroelectrodes were implanted bilaterally in STN and GPi. Therefore, recording of pallidal LFP activity was possible during HFS of STN. We recorded pallidal LFP activity at rest and during three sessions of $200 \mathrm{~s}$ of STN stimulation. $\beta$ activity was consistently suppressed during HFS of the ipsilateral STN (Fig. 9), but no change in pallidal LFP power occurred during contralateral STN HFS (results not shown).

\section{Discussion}

We have shown that $\beta$ activity in the STN LFP is suppressed for several seconds after therapeutic HFS of the STN contralateral to those upper limbs showing a delayed return in bradykinesia after stimulation. The reduction in STN LFP activity was frequency selective and correlated with the degree of per-

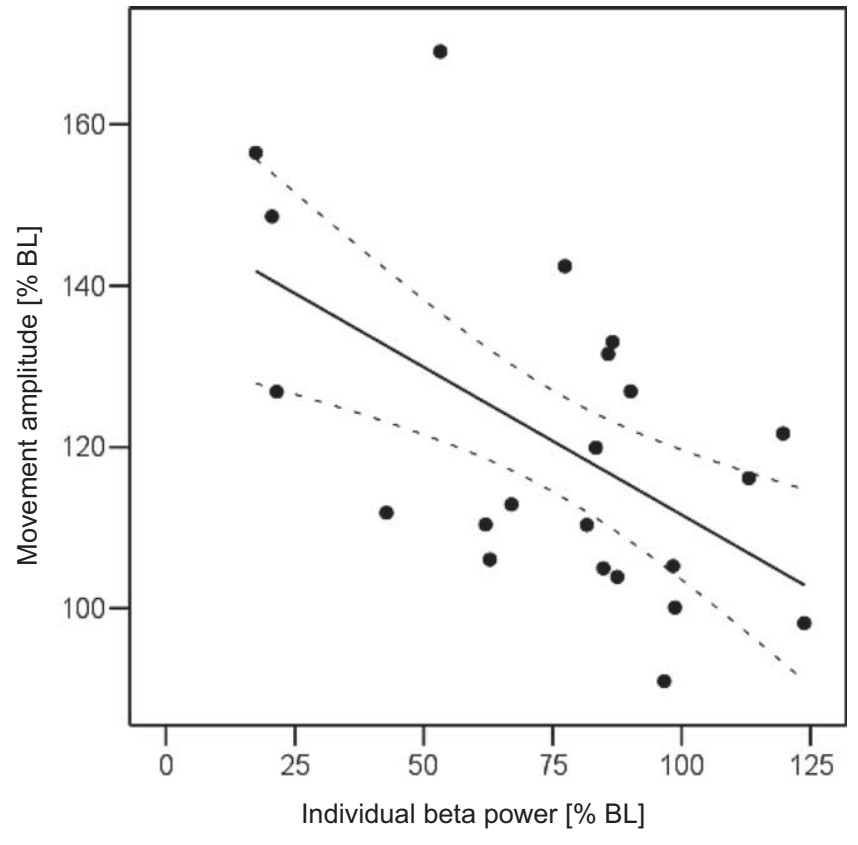

Figure 7. Scatter plot showing the correlation between individual $\beta$ activity (mean STN $\beta$ power over $20 \mathrm{~s}$ before start of the corresponding contralateral hand movement) and movement amplitude (mean RMS movement amplitude over $20 \mathrm{~s}$ ), both expressed as a percentage change from the last (fourth) trial in each session ( $n=11$ sides). The plot includes post-HFS periods 1 and 2 . The significant negative correlation $(r=-0.555 ; p=0.007)$ suggests that reduced $\beta$ activity was associated with better motor performance in the patients. Dotted lines represent $95 \%$ confidence limits.

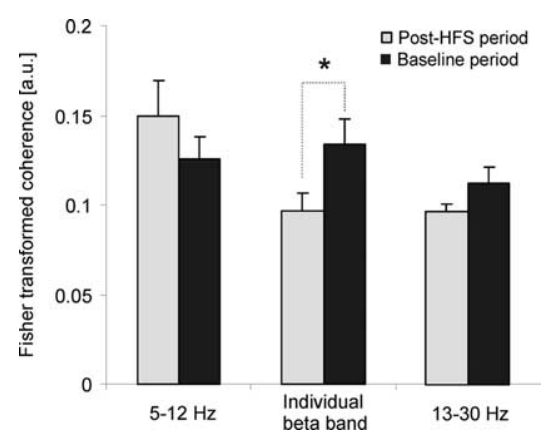

Figure 8. Mean Fisher-transformed STN LFP-EEG coherence values $(n=8)$ for $5-12 \mathrm{~Hz}$, $13-30 \mathrm{~Hz}$, and individual $\beta$ activity over the time periods of $0-12 \mathrm{~s}$ after the end of HFS and during baseline from 128 to $140 \mathrm{~s}$. Note the significant decrease in STN LFP-EEG in the individual $\beta$ band ( $\left.{ }^{*} p<0.05\right)$ after HFS (gray) compared with baseline (black). A similar trend is shown for coherence within the broad $\beta$ band (13-30 Hz; $p=0.06$ ) but not for low-frequency coherence.

sisting improvement in hand movements, supporting a link between HFS-induced improvement in bradykinesia and reduction in $\beta$ activity in the region of the STN in patients with PD. Our findings give correlative evidence that HFS may act by reducing oscillatory STN LFP $\beta$ activity in PD patients, thereby mirroring the effect of levodopa on this activity (Brown et al., 2001; Priori et al., 2004; Kühn et al., 2006). However, the functional consequences of suppression of $\beta$ activity in the STN may not have been confined to the STN area, for cortico-STN coherence in the $\beta$ frequency band was suppressed shortly after HFS, whereas pallidal $\beta$ power was continuously suppressed during STN HFS. Therefore, the reduction in $\beta$ activity in the STN area may be propagated along 


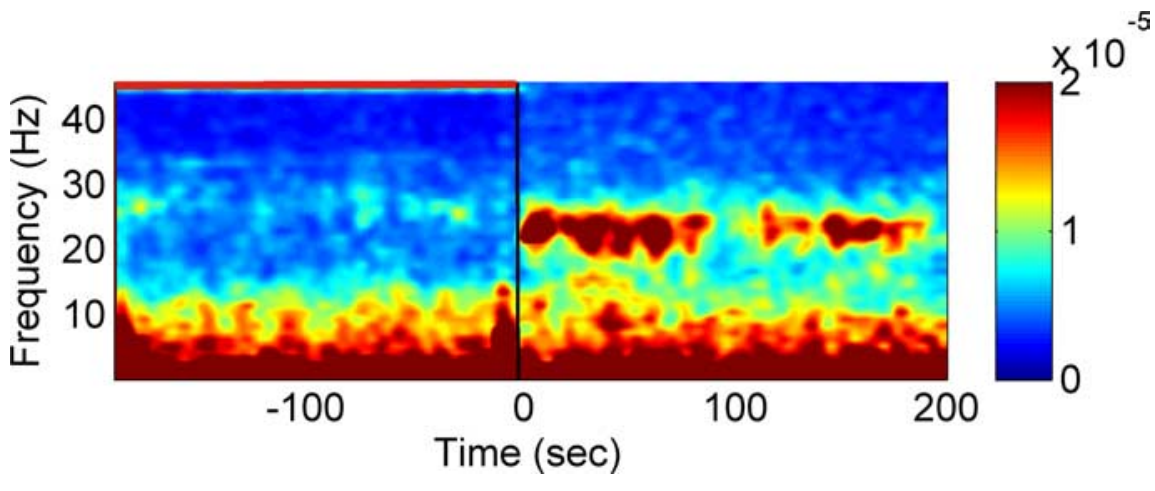

Figure 9. Oscillatory activity recorded from left GPi (contact pair 01) in case 11 during and after bilateral STN HFS. Power is averaged across three sessions of HFS. HFS and rest recordings were performed for $200 \mathrm{~s}$ each (HFS period is indicated by the red horizontal line). Note that $\beta$ power is reduced during STN HFS and recurs shortly after the end of HFS (indicated by the black vertical line). Artifact at $\sim 43 \mathrm{~Hz}$ is a subharmonic of $\mathrm{HFS}$ at $130 \mathrm{~Hz}$.

the different nuclei of the basal ganglia and to the cerebral cortex, so that HFS influences neuronal activity both locally at the site of stimulation and also over other functionally connected elements of the cortex-basal ganglia network. This could arise in two nonmutually exclusive ways. Either the suppression of $\beta$ activity in the STN means that this activity is no longer propagated, or stimulation-induced high-frequency activity of STN neurons (Garcia et al., 2005) is transmitted to the output structures of the basal ganglia (Hashimoto et al., 2003; Maurice et al., 2003), where it further suppresses $\beta$ activity.

Although we recorded STN LFP activity immediately after HFS, it seems likely that a similar suppression of $\beta$ activity also occurs during STN HFS. This is supported by the results in our patient with dual implantation of STN and GPi and those previously reported in another two comparable patients (Brown et al., 2004). Likewise, cortical $\beta$ activity is suppressed during HFS of the STN, with the change correlating with clinical improvement (Silberstein et al., 2005). Levodopa treatment also reduces LFP oscillations in the $\beta$ band in parallel with clinical improvement of motor symptoms (Brown et al., 2001; Priori et al., 2004; Kühn et al., 2006), and a common mechanism of action through $\beta$ suppression might explain why levodopa and HFS correlate in the clinical efficacy achieved (Charles et al., 2002; Welter et al., 2002), but do not have major additive effects (Nutt et al., 2001).

Our findings are consistent with a preliminary report of short-lasting suppression of $\beta$ activity in two PD patients after short periods of HFS performed intraoperatively (Wingeier et al., 2006). Importantly, we also show that HFS-induced suppression of oscillatory activity was frequency specific for the $\beta$ band. Low-frequency $(5-12 \mathrm{~Hz})$ activity did not show significant change after HFS overall, although it could be increased in some patients (for single example, see Fig. 5). The frequency-specific effect of HFS on LFP activity makes it rather unlikely that $\beta$ suppression is caused by stimulationinduced artifacts or saturation of amplifiers after high-voltage inputs during HFS.

One study, however, has failed to show a suppression of LFP $\beta$ activity after HFS (Foffani et al., 2006). There are certain methodological differences between our study and the latter study that may help to explain the contrasting findings. Most importantly, we investigated time-evolving power changes in the frequency range from 1 to $100 \mathrm{~Hz}$ rather then evaluating a fixed time period after cession of HFS. $\beta$ suppression was observed for $\sim 20 \mathrm{~s}$, sim-
$-5 \quad$ ilar to the findings of Wingeier et al. (2006). In the study by Foffani et al. (2006), power was averaged over a period of $60 \mathrm{~s}$, which may have afforded insufficient temporal resolution to pick up more short-lived $\beta$ suppression after HFS. Another critical difference is that we assessed spectral changes in patients with an established delayed return of bradykinesia after discontinuation of HFS. Foffani et al. (2006), in contrast, only formally tested for a delayed return of motor impairment in a small group of patients that were not included in the LFP analysis.

This is not to say that our study did not have its own drawbacks. First, we compared spectral changes after stopping HFS with a baseline period drawn from close to the end of our recording period, 100-140 s after cessation of DBS. Therefore, potential carry-over effects could have led us to underestimate the stimulation-induced effects observed in our study. Indeed, return of clinical deficit may be quite delayed. Temperli et al. (2003) found that motor UPDRS scores were still improved by $\sim 25 \%, 15-30$ min after switching off STN stimulators. It remains to be seen whether this very delayed return in some of the motor impairment also involves reversal of $\beta$ suppression or some other mechanism, perhaps involving plasticity. In addition, we were unable to resolve spectral changes at very low frequency (below $1.5 \mathrm{~Hz}$ ), which may be relevant (Priori et al., 2006), and our study did not address the effect of HFS on rest tremor, where it is likely that other pathophysiological mechanisms are involved (Rivlin-Etzion et al., 2006). Finally, distinction should be made between HFS of basal ganglia targets in PD and the low-frequency $(<40 \mathrm{~Hz})$ stimulation of the pedunculopontine nucleus, which is currently being piloted (Stefani et al., 2007). In the latter case, stimulation is likely to be mimicking a physiological rhythm rather than attenuating a pathological one (Androulidakis et al., 2008).

The HFS-induced effect on $\beta$ activity is similar to that observed after levodopa treatment in PD (Priori et al., 2004) and would be in line with the notion that enhanced $\beta$ activity is antikinetic and may contribute to the pathophysiology of Parkinson's disease (Brown, 2003; Brown and Williams, 2005). Abnormal synchronization of oscillatory LFP activity over $13-30 \mathrm{~Hz}$ has been recorded from basal ganglia nuclei in the 6-hydroxydopamine-lesioned rodent model of PD (Sharott et al., 2005) and PD patients withdrawn from their usual medication (Levy et al., 2002; Priori et al., 2004; Doyle et al., 2005). Furthermore, the degree of suppression of $\beta$ activity by levodopa correlates with the levodopa-induced improvement in motor symptoms (Kühn et al., 2006), the latency of $\beta$ suppression during movement correlates with reaction time in PD (Kühn et al., 2004; Williams et al., 2005), and stimulation at $\beta$ frequency may lead to moderate slowing of movement in patients with relatively preserved motor performance at the time (Chen et al., 2007). Here, we provide further correlative evidence for a link between $\beta$ activity and bradykinesia in PD patients. The significant correlation between the improvement in motor performance and suppression of $\beta$ activity after HFS extends support for the hypothesis that a pathological synchronization of neuronal activity in this frequency range contributes to the pathophysiology of Parkinsonism, specifically bradykinesia, and that reduction in $\beta$ synchrony is therapeutic in PD. The 
therapeutic mechanism of action of HFS may thus be a reduction of aberrant $\beta$ activity.

Our results also have implications for the development of second-generation deep brain stimulators that could potentially be switched on in a demand-controlled way when local $\beta$ activity becomes more pronounced and could be programmed to specifically desynchronize pathologically synchronized activity in the $\beta$ band (Popovych et al., 2006). This mode of operation could prolong battery life and also reduce side effects.

In summary, our study provides new correlative evidence that STN HFS reduces abnormal oscillatory $\beta$ activity in the STN in PD patients that results in a subsequent reduction in coupled $\beta$ activity across the basal ganglia-cortex loop. These findings would further support the hypothesis that a pathological synchronization of neuronal activity in the $\beta$ frequency range may contribute to the pathophysiology of Parkinsonism and that several different treatment options could possibly lead to improvement of PD symptoms by suppression of $\beta$ activity across the cortex-basal ganglia-cortex network.

\section{References}

Androulidakis A, Mazzone P, Litvak V, Penny W, Dileone M, Doyle Gaynor L, Tisch S, Di Lazzaro V, Brown P (2008) Oscillatory activity in the pedunculopontine area of patients with Parkinson's disease. Exp Neurol 27:1277-1284.

Bergman H, Wichmann T, Karmon B, DeLong MR (1994) The primate subthalamic nucleus. II. Neuronal activity in the MPTP model of parkinsonism. J Neurophysiol 72:507-520.

Brillinger DR (1981) Some aspects of modern population mathematics. Can J Stat 9:173-194.

Brown P (2000) Cortical drives to human muscle: the Piper and related rhythms. Prog Neurobiol 60:97-108.

Brown P (2003) Oscillatory nature of human basal ganglia activity: relationship to the pathophysiology of Parkinson's disease. Mov Disord 18:357-363.

Brown P, Eusebio A (2008) Paradoxes of functional neurosurgery: clues from basal ganglia recordings. Mov Disord 23:12-20; quiz 158.

Brown P, Williams D (2005) Basal ganglia local field potential activity: character and functional significance in the human. Clin Neurophysiol 116:2510-2519.

Brown P, Oliviero A, Mazzone P, Insola A, Tonali P, Di Lazzaro V (2001) Dopamine dependency of oscillations between subthalamic nucleus and pallidum in Parkinson's disease. J Neurosci 21:1033-1038.

Brown P, Mazzone P, Oliviero A, Altibrandi MG, Pilato F, Tonali PA, Di Lazzaro V (2004) Effects of stimulation of the subthalamic area on oscillatory pallidal activity in Parkinson's disease. Exp Neurol 188:480-490.

Charles PD, Van Blercom N, Krack P, Lee SL, Xie J, Besson G, Benabid AL, Pollak P (2002) Predictors of effective bilateral subthalamic nucleus stimulation for PD. Neurology 59:932-934.

Chen CC, Litvak V, Gilbertson T, Kühn A, Lu CS, Lee ST, Tsai CH, Tisch S, Limousin P, Hariz M, Brown P (2007) Excessive synchronization of basal ganglia neurons at $20 \mathrm{~Hz}$ slows movement in Parkinson's disease. Exp Neurol 205:214-221.

Doyle LM, Kühn AA, Hariz M, Kupsch A, Schneider GH, Brown P (2005) Levodopa-induced modulation of subthalamic beta oscillations during self-paced movements in patients with Parkinson's disease. Eur J Neurosci 21:1403-1412.

Foffani G, Ardolino G, Egidi M, Caputo E, Bossi B, Priori A (2006) Subthalamic oscillatory activities at beta or higher frequency do not change after high-frequency DBS in Parkinson's disease. Brain Res Bull 69:123-130.

Garcia L, D'Alessandro G, Bioulac B, Hammond C (2005) High-frequency stimulation in Parkinson's disease: more or less? Trends Neurosci 28:209-216.

Gatev P, Darbin O, Wichmann T (2006) Oscillations in the basal ganglia under normal conditions and in movement disorders. Mov Disord 21:1566-1577.

Halliday DM, Rosenberg JR, Amjad AM, Breeze P, Conway BA, Farmer SF
(1995) A framework for the analysis of mixed time series/point process data-theory and application to the study of physiological tremor, single motor unit discharges and electromyograms. Prog Biophys Mol Biol 64:237-278.

Hammond C, Bergman H, Brown P (2007) Pathological synchronization in Parkinson's disease: networks, models and treatments. Trends Neurosci 30:357-364.

Hariz MI, Krack P, Melvill R, Jorgensen JV, Hamel W, Hirabayashi H, Lenders M, Wesslen N, Tengvar M, Yousry TA (2003) A quick and universal method for stereotactic visualization of the subthalamic nucleus before and after implantation of deep brain stimulation electrodes. Stereotact Funct Neurosurg 80:96-101.

Hashimoto T, Elder CM, Okun MS, Patrick SK, Vitek JL (2003) Stimulation of the subthalamic nucleus changes the firing pattern of pallidal neurons. J Neurosci 23:1916-1923.

Kempf F, Kühn AA, Kupsch A, Brücke C, Weise L, Schneider GH, Brown P (2007) Premovement activities in the subthalamic area of patients with Parkinson's disease and their dependence on task. Eur J Neurosci 25:3137-3145.

Kühn AA, Williams D, Kupsch A, Limousin P, Hariz M, Schneider GH, Yarrow K, Brown P (2004) Event-related beta desynchronization in human subthalamic nucleus correlates with motor performance. Brain 127:735-746.

Kühn AA, Trottenberg T, Kivi A, Kupsch A, Schneider GH, Brown P (2005) The relationship between local field potential and neuronal discharge in the subthalamic nucleus of patients with Parkinson's disease. Exp Neurol 194:212-220.

Kühn AA, Kupsch A, Schneider GH, Brown P (2006) Reduction in subthalamic 8-35 Hz oscillatory activity correlates with clinical improvement in Parkinson's disease. Eur J Neurosci 23:1956-1960.

Levy R, Hutchison WD, Lozano AM, Dostrovsky JO (2002) Synchronized neuronal discharge in the basal ganglia of parkinsonian patients is limited to oscillatory activity. J Neurosci 22:2855-2861.

Maurice N, Thierry AM, Glowinski J, Deniau JM (2003) Spontaneous and evoked activity of substantia nigra pars reticulata neurons during highfrequency stimulation of the subthalamic nucleus. J Neurosci 23:9929-9936.

Meissner W, Leblois A, Hansel D, Bioulac B, Gross CE, Benazzouz A, Boraud T (2005) Subthalamic high frequency stimulation resets subthalamic firing and reduces abnormal oscillations. Brain 128:2372-2382.

Nini A, Feingold A, Slovin H, Bergman H (1995) Neurons in the globus pallidus do not show correlated activity in the normal monkey, but phaselocked oscillations appear in the MPTP model of parkinsonism. J Neurophysiol 74:1800-1805.

Nutt JG, Rufener SL, Carter JH, Anderson VC, Pahwa R, Hammerstad JP, Burchiel KJ (2001) Interactions between deep brain stimulation and levodopa in Parkinson's disease. Neurology 57:1835-1842.

Popovych OV, Hauptmann C, Tass PA (2006) Control of neuronal synchrony by nonlinear delayed feedback. Biol Cybern 95:69-85.

Priori A, Foffani G, Pesenti A, Tamma F, Bianchi AM, Pellegrini M, Locatelli M, Moxon KA, Villani RM (2004) Rhythm-specific pharmacological modulation of subthalamic activity in Parkinson's disease. Exp Neurol 189:369-379.

Priori A, Ardolino G, Marceglia S, Mrakic-Sposta S, Locatelli M, Tamma F, Rossi L, Foffani G (2006) Low-frequency subthalamic oscillations increase after deep brain stimulation in Parkinson's disease. Brain Res Bull 71:149-154.

Rivlin-Etzion M, Marmor O, Heimer G, Raz A, Nini A, Bergman H (2006) Basal ganglia oscillations and pathophysiology of movement disorders. Curr Opin Neurobiol 16:629-637.

Sharott A, Magill PJ, Harnack D, Kupsch A, Meissner W, Brown P (2005) Dopamine depletion increases the power and coherence of betaoscillations in the cerebral cortex and subthalamic nucleus of the awake rat. Eur J Neurosci 21:1413-1422.

Silberstein P, Pogosyan A, Kühn AA, Hotton G, Tisch S, Kupsch A, Dowsey-Limousin P, Hariz MI, Brown P (2005) Cortico-cortical coupling in Parkinson's disease and its modulation by therapy. Brain 128:1277-1291.

Stefani A, Lozano AM, Peppe A, Stanzione P, Galati S, Tropepi D, Pierantozzi M, Brusa L, Scarnati E, Mazzone P (2007) Bilateral deep brain stimulation of the pedunculopontine and subthalamic nuclei in severe Parkinson's disease. Brain 130:1596-1607. 
Temperli P, Ghika J, Villemure JG, Burkhard PR, Bogousslavsky J, Vingerhoets FJ (2003) How do parkinsonian signs return after discontinuation of subthalamic DBS? Neurology 60:78-81.

Uhlhaas PJ, Singer W (2006) Neural synchrony in brain disorders: relevance for cognitive dysfunctions and pathophysiology. Neuron 52:155-168.

Volkmann J, Moro E, Pahwa R (2006) Basic algorithms for the programming of deep brain stimulation in Parkinson's disease. Mov Disord 21 [Suppl 14]:S284-S289.

Weinberger M, Mahant N, Hutchison WD, Lozano AM, Moro E, Hodaie M, Lang AE, Dostrovsky JO (2006) Beta oscillatory activity in the subthalamic nucleus and its relation to dopaminergic response in Parkinson's disease. J Neurophysiol 96:3248-3256.
Welter ML, Houeto JL, Tezenas du Montcel S, Mesnage V, Bonnet AM, Pillon B, Arnulf I, Pidoux B, Dormont D, Cornu P, Agid Y (2002) Clinical predictive factors of subthalamic stimulation in Parkinson's disease. Brain 125:575-583.

Williams D, Kühn A, Kupsch A, Tijssen M, van Bruggen G, Speelman H, Hotton G, Loukas C, Brown P (2005) The relationship between oscillatory activity and motor reaction time in the parkinsonian subthalamic nucleus. Eur J Neurosci 21:249-258.

Wingeier B, Tcheng T, Koop MM, Hill BC, Heit G, Bronte-Stewart HM (2006) Intra-operative STN DBS attenuates the prominent beta rhythm in the STN in Parkinson's disease. Exp Neurol 197:244251. 